IAU Colloquium 164: Radio Emission from Galactic and Extragalactic Compact Sources ASP Conference Series, Vol. 144, 1998

J. A. Zensus, G. B. Taylor, \& J. M. Wrobel (eds.)

\title{
Multi-Frequency VLBI Observations of the GPS Source 1934-638
}

A. K. Tzioumis, E. A. King, J. E. Reynolds, D. L. Jauncey, \& R. G. Gough Australia Telescope National Facility, Australia

R. A. Preston, D. W. Murphy, S. J. Tingay, D. L. Meier, \& D. L. Jones

Jet Propulsion Laboratory, Pasadena, CA, USA

J. E. J. Lovell \& P. G. Edwards

Institute of Space and Astronautical Science, Japan

P. M. McCulloch \& M. E. Costa

University of Tasmania, Hobart, Australia

L. Kedziora-Chudczer \& D. Campbell-Wilson

University of Sydney, Sydney, Australia

G. D. Nicolson \& J. F. H. Quick

Hartebeesthoek Radio Astronomy Observatory, Krugersdorp, South Africa

Abstract. PKS 1934-638 is an archetypal GPS source, peaking at $1.4 \mathrm{GHz}$ and exhibits almost no flux density variability. VLBI images at frequencies of $.843,2.3,4.8, \& 8.4$ were made with the southern hemisphere VLBI array and they reveal that the source is a 42 mas compact double. There is no detectable change in separation over the last 20 years, yielding an upper limit of $\sim 0.03 c \pm 0.2 c$ on any expansion velocity. The spectral shapes of the two components are remarkably similar, despite indications of finer structure on longer baselines. Magnetic field calculations indicate fields of a few mGauss and the results are consistent with equipartition.

PKS 1934-638 has been identified with an $18.9^{m}$ galaxy (Kellermann 1966) at a redshift of 0.183 (Penston \& Fosbury 1978). Deep CCD images of this galaxy show two compact galaxies within $2.9^{\prime \prime}$, sharing a common envelope, and with the radio source located within $0.1^{\prime \prime}$ of the brighter galaxy (Jauncey et al. 1986). Optical spectroscopic observations (Fosbury et al. 1987) measure very high line ratios, indicating larger amounts of gas and dust than found in typical narrow line radio galaxies.

As is evident in Figure 1 (LHS), PKS 1934-638 shows a very sharply peaked radio spectrum at $1.4 \mathrm{GHz}$, first noted by Bolton, Gardner, \& Mackey (1963). The radio flux density has been extensively monitored for over 30 years at Parkes, Hartebeesthoek, Hobart, and the Australia Telescope Compact Array (ATCA) and shows little or no variability. Thus, PKS 1934-638 has become the primary flux calibrator for the ATCA. There is no detected radio structure at scales $\geq 1^{\prime \prime}$ and no linear polarization $(\leq 0.1 \%)$.

Early VLBI observations (Gubbay et al. 1971; Tzioumis et al. 1989) at $2.3 \mathrm{GHz}$, indicated that the source is a compact double with a separation of $42.0 \pm 0.2$ mas. Recent (1988-92) VLBI observations were made with the Southern Hemisphere network (Preston et al. 1989) at 2.3,4.8, and $8.4 \mathrm{GHz}$ (King 1994) with approximately the same resolution. The source appears remarkably similar at these frequencies and the $4.8 \mathrm{GHz}$ image is presented in Figure 1 (RHS). More than $90 \%$ of the total flux density was detected at all frequencies, implying that there is little or no extended structure. About $15 \%$ of the total flux density is detected on intercontinental baselines (Australia to 

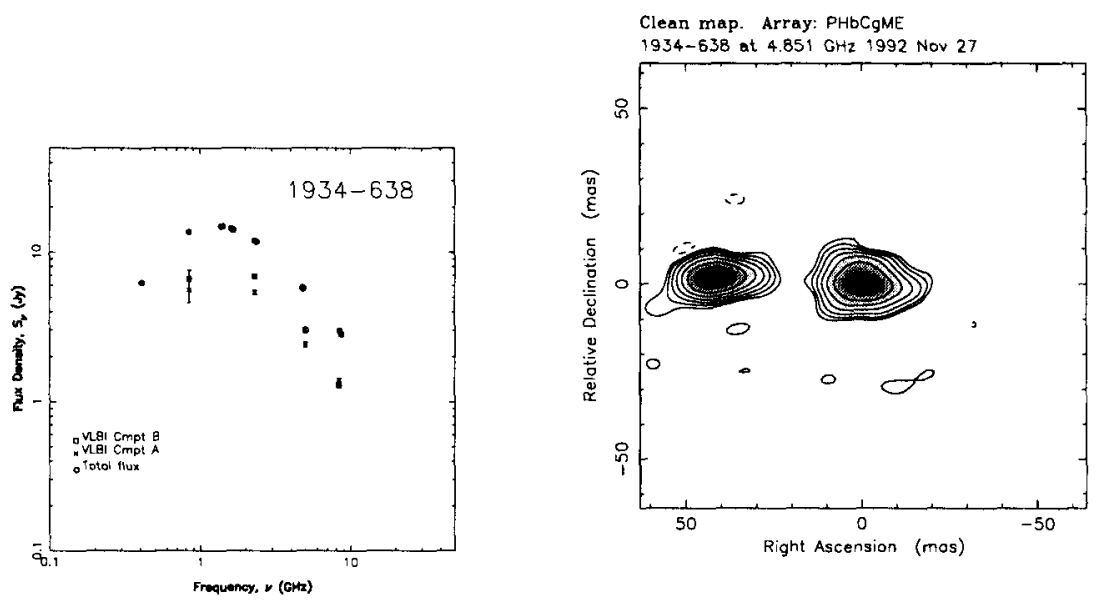

Figure 1. LHS: The power spectra of PKS 1934-638 and its components between .408 and $8.4 \mathrm{GHz}$. The total flux density (top set of points) is a compilation from published results and the component spectra (lower 2 sets of points) are measurements from VLBI images. RHS: VLBI image of PKS 1934-638 at $4.8 \mathrm{GHz}$. The peak flux density is $2.1 J_{y} . b e a m^{-1}$ and the contours are at $-0.75,0.75,1.5,3,6,12,24,48,96 \%$ (Beam FWHM 10x5.5 mas, $P A=-86^{\circ}$ ).

South Africa) and the visibilities beat rapidly indicating the presence of very compact components (King 1994). VLBI observations at $843 \mathrm{MHz}$ (Parkes, Hobart, MOST) barely resolve the source (beam $40 \times 77$ mas), but a consistent double component model can be fitted.

These multi-frequency VLBI observations allow the determination of the spectral shapes of the compact components, as shown in Figure 1 (LHS). Both component spectra are very similar and peak near $1.4 \mathrm{GHz}$. Magnetic field calculations using equipartition arguments indicate $B_{m e} \sim 5 \times 10^{-3}$ Gauss, and similarly from synchrotron self-absorption $B_{s s a} \sim 1-3 \times 10^{-3}$ Gauss. Within the uncertainties inherent in these calculations, these results indicate that the components of PKS 1934-638 are consistent with equipartition.

No change in component separation has been detected with VLBI observations spanning over 20 years. Thus there is little or no expansion of this radio source, with an upper limit on expansion velocity of $\sim 0.03 c \pm 0.2 c$.

\section{References}

Bolton, J. G., Gardner, F. F., \& Mackey, M. B. 1963. Nature, 199, 682-683.

Fosbury, R. A. E., et al. 1987. MNRAS, 225, 761-768.

Gubbay, J. S., et al. 1971. $A J$, 76, 965-969.

Jauncey, D. L., et al. 1986. $A J, 92,1036-1038$.

Kellermann, K. I. 1966. Aust. J. Phys. 19, 195-207.

King, E. A. 1994. Ph.D. Thesis, University of Tasmania.

Penston, M. V., \& Fosbury, R. A. E. 1978. MNRAS, 183, 479-490.

Preston, R. A., et al. 1989. $A J$, 98, 1-26.

Tzioumis, A. K., et al. 1989. $A J, 98,36-43$. 